\title{
17. COCCOLITH AGE DETERMINATIONS LEG 4, DEEP SEA DRILLING PROJECT
}

\author{
David Bukry, U. S. Geological Survey, La Jolla ${ }^{1}$
}

Leg 4 of the Deep Sea Drilling Project, FebruaryMarch 1969, recovered 120 cores. A total of 173 samples from these cores were examined by lightmicroscope techniques for coccolith assemblages. Agediagnostic species in selected samples from each drilling site, listed by biostratigraphic zone, follow a brief summary of the coccolith stratigraphy at each drilling site. The coccolith zones used in this report are those described by Bukry and Bramlette (1970) in the report on Leg 3. During study of Leg 4 material, M. N. Bramlette, of Scripps Institution of Oceanography, provided valuable information on the stratigraphic assignment of several of these cores.

Sample numbers given under the biostratigraphic zones for each site represent, in the following sequence: (cruise-leg number)-(drill-hole designation, consisting of site number, plus a letter if more than one hole)(core designation)-(core-section number). This series is followed by the interval below the top of each core section in centimeters. For example, 4-27A-1 A-1, $75-76 \mathrm{~cm}$, indicates that the sample came from Leg 4 , Hole 27A (at Site 27), the first barrel of core recovered, the top section of that core, and from 75 to 76 centimeters below the top of the section. Most core runs were 9.1 meters long, but commonly the core liners were not full. In this report, recoveries are arbitrarily placed at the top of the core runs, and an approximate depth in meters below the sea floor follows each sample number.

Species considered in this report are listed below in Table 1.

TABLE 1

Coccolith Species Considered in This Report

Bramletteius serraculoides Gartner

Campylosphaera dela (Bramlette and Sullivan)

Catinaster calyculus Martini and Bramlette

Ceratolithus cristatus Kamptner

Ceratolithus rugosus Bukry and Bramlette

Ceratolithus tricorniculatus Gartner

\footnotetext{
${ }^{1}$ Publication authorized by the Director, U.S. Geological Survey.
}

TABLE 1 - Continued

Chiphragmalithus austriacus (Stradner)

Original designation:

Trochoaster austriacus Stradner, 1959. Proc. Fifth

World Petrol. Congr. 1, 1088 (Figure 11).

Chiphragmalithus mexicanus (Stradner)

Original designation:

Trochoaster mexicanus Stradner, 1959. Erdoel-

Zeitschrift. 75, 480 (Figure 55).

Chiphragmalithus sp.

Chiasmolithus gigas (Bramlette and Sullivan)

Original designation:

Coccolithus gigas Bramlette and Sullivan, 1961. Micropaleontology. 7 (2), 140 (Plate 1, Figures 6a6d).

Chiasmolithus grandis (Bramlette and Riedel)

Original designation:

Coccolithus grandis Bramlette and Riedel, 1954. J. Paleóntol. 28 (4), 391 (Plate 38, Figures 1a-1b).

Chiasmolithus staurion (Bramlette and Sullivan) Original designation:

Coccolithus staurion Bramlette and Sullivan, 1961. Micropaleontology. 7 (2), 141 (Plate 2, Figures 5a$5 \mathrm{~b}, 6 \mathrm{a}-6 \mathrm{c})$.

Chiasmolithus sp.

Coccolithus bisectus (Hay, Mohler, and Wade) of Bramlette and Wilcoxon

Coccolithus sp. aff. C. bisectus (Hay, Mohler, and Wade) of Bramlette and Wilcoxon

Coccolithus doronicoides Black and Barnes

Coccolithus eopelagicus (Bramlette and Riedel)

Cyclococcolithus leptoporus (Murray and Blackman) varieties A, B, and C of McIntyre, Bé, and Preikstas

Cyclococcolithus lusitanicus (Black)

Cyclococcolithus neogammation Bramlette and Wilcoxon

Cyclolithella annula (Cohen)

Discoaster barbadiensis Tan

Discoaster bollii Martini and Bramlette

Discoaster sp. aff. D. bollii Martini and Bramlette

Discoaster brouweri Tan 


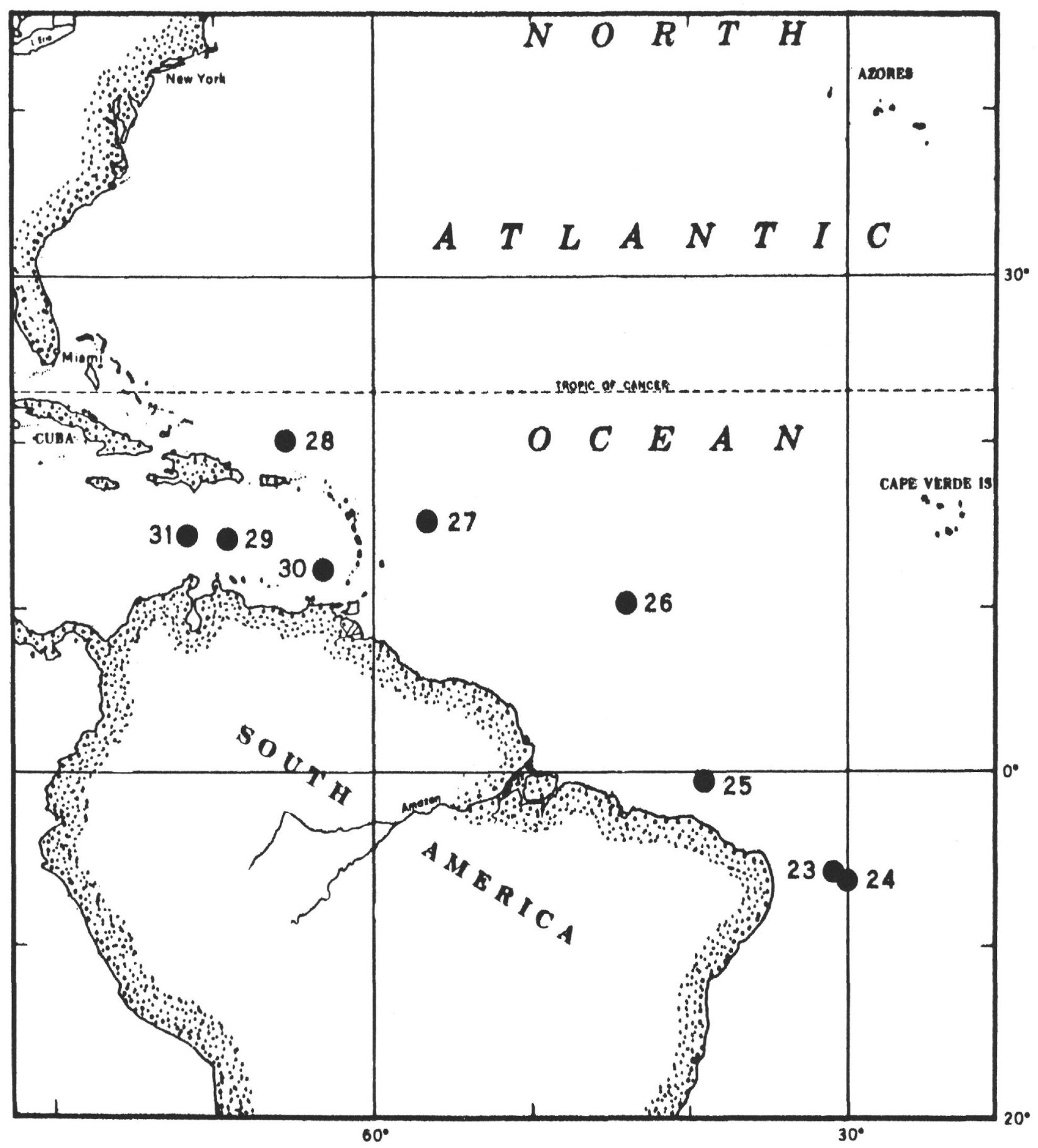

Figure 1. Sites drilled on Leg 4, Deep Sea Drilling Project. 
TABLE 1 - Continued

Discoaster sp. aff. D. brouweri Tan

Discoaster calcaris Gartner

Discoaster challengeri Bramlette and Riedel

Discoaster sp. aff. D. colleti (Parejas)

Discoaster deflandrei Bramlette and Riedel

Discoaster distinctus Martini

Discoaster exilis Martini and Bramlette

Discoaster hamatus Martini and Bramlette

Discoaster sp. aff. D. hamatus Martini and Bramlette

Discoaster lodoensis Bramlette and Riedel

Discoaster pentaradiatus Tan

Discoaster perclarus Hay

Discoaster perplexus Bramlette and Riedel

Discoaster saipanensis Bramlette and Riedel

Discoaster sublodoensis Bramlette and Sullivan

Discoaster subsurculus Gartner

Discoaster surculus Martini and Bramlette

Discoaster tani nodifer Bramlette and Riedel

Discoaster variabilis Martini and Bramlette

Discoasteroides kuepperi (Stradner)

Gephyrocapsa oceanica Kamptner

Helicopontosphaera ampliaperta (Bramlette and Wilcoxon)

Original designation:

Helicosphaera ampliaperta Bramlette and Wilcoxon, 1967. Tulane Stud. Geol. 5 (3), 105 (Plate 6, Figures 1-4).

\section{Helicopontosphaera intermedia (Martini)}

Helicopontosphaera kamptneri Hay and Mohler

Helicopontosphaera parallela (Bramlette and Wilcoxon) Original designation:

Helicosphaera parallela Bramlette and Wilcoxon, 1967. Tulane Stud. Geol. 5 (3), 106 (Plate 5, Figures 9-10).

Helicopontosphaera sp. aff. H. seminulum (Bramlette and Sullivan)

Original designation:

Helicosphaera seminulum seminulum Bramlette and Sullivan, 1961. Micropaleontology. 7 (2), 144, Plate 4, Figures 1a-1c, 2.

Helicopontosphaera truncata (Bramlette and Wilcoxon) Original designation:

Helicosphaera truncata Bramlette and Wilcoxon, 1967. Tulane Studies Geol. 5 (3), 106 (Plate 6, Figures 13-14).
TABLE 1 - Continued

Oolithotus antillarum (Cohen)

Reticulofenestra dictyoda (Deflandre)

Reticulofenestra pseudoumbilica (Gartner)

Reticulofenestra umbilica (Levin)

Rhabdosphaera clavigera Murray and Blackman

Rhabdosphaera stylifera Lohmann

Scapholithus fossilis Deflandre

Scapholithus sp. aff. S. fossilis Deflandre

Sphenolithus abies Deflandre

Sphenolithus sp. aff. S. belemnos Bramlette and Wilcoxon

Sphenolithus ciperoensis Bramlette and Wilcoxon

Sphenolithus furcatolithoides Locker

Sphenolithus heteromorphus Deflandre

Sphenolithus moriformis (Brönnimann and Stradner)

Sphenolithus predistentus Bramlette and Wilcoxon

Sphenolithus radians Deflandre

Triquetrorhabdulus carinatus Martini

Triquetrorhabdulus rugosus Bramlette and Wilcoxon

Triquetrorhabdulus sp. aff. T. rugosus Bramlette and Wilcoxon

Triquetrorhabdulus sp.

Umbilicosphaera mirabilis Lohmann

Zygolithus dubius Deflandre

\section{HOLE 23}

(lat $6^{\circ} 08.75^{\prime} \mathrm{S}$., long $31^{\circ} 02.60^{\prime} \mathrm{W}$., depth 5079 meters)

\section{Summary of Coccolith Age Determinations}

The lower Pleistocene sample at 5 meters contains rare specimens of discoasters and Gephyrocapsa oceanica. In Cores 5 and 6 the lower part of the Miocene to Oligocene Triquetrorhabdulus carinatus Zone is represented by generally poor assemblages. This is the oldest sediment sampled. Most samples from this hole do not contain coccoliths.

Age-diagnostic Coccoliths in Selected Samples, Hole 23

$$
\begin{gathered}
\text { Lower Pleistocene } \\
\text { (Coccolithus doronicoides Zone) }
\end{gathered}
$$

4-23-1-4, $94 \mathrm{~cm}$, depth $5 \mathrm{~m}$ :

Ceratolithus cristatus, Coccolithus doronicoides, Cyclococcolithus leptoporus vars. A and B, Cyclolithella annula, Rhabdosphaera clavigera. 


\section{Miocene or Oligocene \\ (Triquetrorhabdulus carinatus Zone)}

4-23-5-1, $26 \mathrm{~cm}$, depth $121 \mathrm{~m}$ :

Chiasmolithus sp., Coccolithus eopelagicus, Cyclococcolithus neogammation, Discoaster deflandrei, Helicopontosphaera intermedia, Helicopontosphaera kamptneri, Helicopontosphaera parallela, Helicopontosphaera truncata, Sphenolithus sp. aff. S. belemnos, Triquetrorhabdulus carinatus.

\section{HOLE 24}

(lat $6^{\circ} 16.30^{\prime}$ S., long $30^{\circ} 53.53^{\prime} W$., depth 5148 meters)

\section{Summary of Coccolith Age Determinations}

The shallowest sample from this site contains coccoliths of the Triquetrorhabdulus carinatus Zone, Miocene to Oligocene. Deeper samples contain only rare specimens of Coccolithus bisectus, Cyclococcolithus neogammation, and Sphenolithus moriformis, indicating an Oligocene age. Since all of these coccoliths are generally resistant to destruction, this assemblage indicates solution effects and possible reworking.

Age-diagnostic Coccoliths in Selected Samples, Hole 24

Miocene or Oligocene

(Triquetrorhabdulus carinatus Zone)

4-24-1-1, $35 \mathrm{~cm}$, depth $198 \mathrm{~m}$ :

Coccolithus sp. aff. C. bisectus, Cyclococcolithus neogammation, Discoaster deflandrei [5- and 6-rayed], Helicopontosphaera intermedia, Sphenolithus sp. aff. $S$. belemnos, Triquetrorhabdulus carinatus.

HOLES 25 AND 25A

(lat $0^{\circ} 31.00^{\prime} S$., long $39^{\circ} 14.40^{\prime} \mathrm{W}$., depth 1916 meters)

\section{Summary of Coccolith Age Determinations}

Samples examined from this site contain well-preserved coccolith assemblages representing the upper Pleistocene to lower Miocene. Samples from the upper cores (Cores 1 and 2) are Pleistocene, and those from the lower ones, Cores 3,4, 5 and 1A, are Miocene. The Miocene section is mainly lower upper Miocene. The redrilling of the site at Hole 25A succeeded in recovering an upper middle to lower upper Miocene sequence.

Age-diagnostic Coccoliths in Selected Samples, Hole 25

$$
\begin{gathered}
\text { Upper Pleistocene } \\
\text { (Gephyrocapsa oceanica Zone) }
\end{gathered}
$$

4-25-1-1, $110 \mathrm{~cm}$, depth $1 \mathrm{~m}$ :

Cyclococcolithus leptoporus var. B, Discoaster perplexus, Gephyrocapsa oceanica [few], Oolithotus antillarum, Scapholithus sp. [common], Umbilicosphaera mirabilis.

\section{Lower Pleistocene \\ (Coccolithus doronicoides Zone)}

4-25-2-3, 0-2 cm, depth $12 \mathrm{~m}$ :

Ceratolithus rugosus, Coccolithus doronicoides, Cyclococcolithus leptoporus vars. A and B, Discoaster perplexus, Rhabdosphaera clavigera, Rhabdosphaera stylifera, Scapholithus sp.

\section{Upper Miocene \\ (Ceratolithus tricorniculatus Zone)}

4-25-3-1, $150 \mathrm{~cm}$, depth $20 \mathrm{~m}$ :

Ceratolithus tricorniculatus, Discoaster brouweri. Discoaster challengeri, Discoaster pentaradiatus, Discoaster surculus.

\section{Lower Upper Miocene}

(Triquetrorhabdulus rugosus Zone)

4-25-4-2, 0-2 cm, depth $29 \mathrm{~m}$ :

Discoaster challengeri, Discoaster sp. aff. D. hamatus, Discoaster perclarus, Discoaster perplexus, Sphenolithus abies, Triquetrorhabdulus rugosus.

Lower Miocene

(Sphenolithus heteromorphus Zone)

4-25-5-CC, depth $46 \mathrm{~m}$ :

Cyclococcolithus leptoporus, Cyclococcolithus neogammation, Discoaster brouweri, Discoaster challengeri, Discoaster deflandrei, Discoaster exilis, Reticulofenestra pseudoumbilica, Sphenolithus heteromorphus.

Taxa representing contamination or reworking from a probable lower Oligocene source: Coccolithus bisectus, Cyclococcolithus lusitanicus, Reticulofenestra umbilica, Sphenolithus predistentus.

Age-diagnostic Coccoliths in Selected Samples, Hole 25A

\section{Lower Upper Miocene \\ (Triquetrorhabdulus rugosus Zone)}

4-25A-1A-1, 75-76 cm, depth $50 \mathrm{~m}$ :

Discoaster challengeri, Discoaster sp. aff. D. hamatus, Discoaster pentaradiatus, Reticulofenestra pseudoumbilica, Sphenolithus abies, Triquetrorhabdulus rugosus.

$$
\begin{aligned}
& \text { Upper Middle Miocene } \\
& \text { (Discoaster hamatus Zone) }
\end{aligned}
$$

4-25A-1A-2, 148-150 cm, depth $52 \mathrm{~m}$ :

Catinaster calyculus, Discoaster challengeri, Discoaster hamatus, Reticulofenestra pseudoumbilica, Sphenolithus abies, Triquetrorhabdulus rugosus. 
HOLE 26

(lat $10^{\circ} 53.55^{\prime} \mathrm{N}$., long $44^{\circ} 02.57^{\prime} \mathrm{W}$., depth 5169 meters)

\section{Summary of Coccolith Age Determinations}

Samples from Cores 1, 2, 3, 4 and 5 from this site all contain sparse Pleistocene coccolith assemblages of the Gephyrocapsa oceanica Zone. All of the assemblages are greatly diluted by nonorganic, siliceous detritus. Rapid sedimentation from Amazon River outflow and dissolving of calcite fossils at depth accounts for the unusually sparse assemblages in this tropical, openocean depositional environment.

Age-diagnostic Coccoliths in Selected Samples, Hole 26

\section{Pleistocene \\ (Gephyrocapsa oceanica Zone)}

4-26-1-1, 148-150 cm, depth $98 \mathrm{~m}$ :

Gephyrocapsa oceanica, Helicopontosphaera kamptneri.

4-26, center-bit sample at $229 \mathrm{~m}$ :

Gephyrocapsa oceanica, Helicopontosphaera kamptneri.

\section{HOLES 27 AND 27A \\ (lat $15^{\circ} 51.39^{\prime} \mathrm{N}$., long $56^{\circ} 52.76^{\prime} \mathrm{W}$., depth 5251 meters)}

\section{Summary of Coccolith Age Determinations}

In Hole 27 at this site, seven cores were recovered. No coccoliths are present in the upper Cores 1, 2, 3 and 4. Core 5, however, contains excellent upper Oligocene assemblages of the Sphenolithus ciperoensis Zone. In Cores 6 and 7, middle Eocene assemblages of the upper part of the Reticulofenestra umbilica Zone are present. The coccoliths in these two lowermost cores appear to be slightly decalcified. Samples from a redrilling of this site at Hole $27 \mathrm{~A}$ are all barren of coccoliths.

Age-diagnostic Coccoliths in Selected Samples, Hole 27

Upper Oligocene

(Sphenolithus ciperoensis Zone)

4-27-5-3, 75-76 cm, depth $373 \mathrm{~m}$ :

Coccolithus bisectus, Coccolithus sp. aff. C. bisectus, Cyclococcolithus neogammation, Helicopontosphaera parallela, Helicopontosphaera sp. aff. H. seminulum, Helicopontosphaera truncata, Sphenolithus ciperoensis, Sphenolithus moriformis, Triquetrorhabdulus carinatus.

\section{Middle Eocene \\ (Reticulofenestra umbilica Zone)}

4-27-7-1, $10 \mathrm{~cm}$, depth $474 \mathrm{~m}$ :

Chiasmolithus grandis, Cyclococcolithus lusitanicus, Discoaster barbadiensis, Discoaster saipanensis, Discoaster tani nodifera, Reticulofenestra umbilica, Sphenolithus furcatolithoides.
HOLE 28

(lat $20^{\circ} 35.19^{\prime} \mathrm{N}$., long $65^{\circ} 37.33^{\prime} \mathrm{W}$., depth 5521 meters)

\section{Summary of Coccolith Age Determinations}

Coccoliths are present in Cores 3 and 5. In Core 3 the coccolith assemblages indicate the middle Eocene Chiphragmalithus quadratus Zone; and, in the Core 5 core catcher, the lower middle Eocene Discoaster sublodoensis Zone. A large variety of species compose these assemblages.

Age-diagnostic Coccoliths in Selected Samples, Hole 28

Middle Eocene

(Chiphragmalithus quadratus Zone)

4-28-3-6, 71-72 cm, depth $176 \mathrm{~m}$ :

Bramletteius serraculoides, Campylosphaera dela, Chiasmolithus grandis, Coccolithus eopelagicus, Discoaster barbadiensis, Discoaster distinctus, Discoaster saipanensis, Reticulofenestra umbilica [small], Sphenolithus furcatolithoides, Zygolithus dubius.

\section{Middle Eocene \\ (Discoaster sublodoensis Zone)}

4-28-5, core catcher, depth $245 \mathrm{~m}$ : Braarudosphaera discula, Campylosphaera dela, Chiasmolithus gigas, Chiasmolithus grandis, Chiasmolithus staurion, Chiphragmalithus sp., Discoaster barbadiensis, Discoaster lodoensis, Discoaster saipanensis, Discoaster sublodoensis, Reticulofenestra dictyoda, Sphenolithus radians, Triquetrorhabdulus sp.

HOLES 29, 29A, AND 29B

(lat $14^{\circ} 47.11^{\prime} \mathrm{N}$., long $69^{\circ} 19.36^{\prime} \mathrm{W}$., depth 4247 meters)

Summary of Coccolith Age Determinations

Sediment recovered from Hole 29 (18 cores) contains coccolith assemblages ranging from upper Pleistocene (Gephyrocapsa oceanica Zone) to the lower middle Eocene (Discoaster sublodoensis Zone). Good coccolith assemblages are present in Cores 1 to 4 ; however, siliceous microfossil ooze dominates in Cores 8 to 18 .

Samples from Hole 29A are barren of coccoliths.

Sediment recovered from Hole 29B (10 cores) contains coccolith assemblages ranging from lower upper Miocene (Triquetrorhabdulus rugosus Zone) to lower middle (?) Eocene (?Discoaster sublodoensis Zone). Most of the coccolith samples (Cores 4B, 5B, 6B) are upper lower Miocene (Helicopontosphaera ampliaperta Zone). Siliceous microfossils dominate the sediment below the level of Core 6B. Eocene coccoliths occur with this sediment type in Core 9B. 
Age-diagnostic Coccoliths in Selected Samples, Hole 29

Upper Pleistocene

(Gephyrocapsa oceanica Zone)

4-29-1-1, 75-76 cm, depth $1 \mathrm{~m}$ :

Gephyrocapsa oceanica, Helicopontosphaera kamptneri.

\section{Upper Pliocene \\ (Discoaster brouweri Zone)}

4-29-4-1, 94-95 cm, depth $28 \mathrm{~m}$ :

Ceratolithus cristatus, Cyclococcolithus leptoporus var. A, Discoaster brouweri, Discoaster pentaradiatus, Discoaster surculus.

Lower Miocene

(?Helicopontosphaera ampliaperta Zone)

4-29-7-1, 106-107 cm, depth $118 \mathrm{~m}$ :

Coccolithus sp. aff. C. bisectus, Cyclococcolithus neogammation, Discoaster deflandrei, Sphenolithus heteromorphus [few], Sphenolithus moriformis, Triquetrorhabdulus carinatus.

Lower Miocene and Upper Eocene, Mixed

4-29-9-2, 76-77 cm, depth $137 \mathrm{~m}$ :

Lower Miocene taxa. Cyclococcolithus neogammation, Discoaster challengeri, Discoaster deflandrei, Sphenolithus sp. aff. S. belemnos [no stem], Sphenolithus heteromorphus.

Upper Eocene taxa. Chiasmolithus sp. [rims], Discoaster barbadiensis, Reticulofenestra umbilica.

\section{Middle Eocene \\ (Chiphragmalithus quadratus Zone)}

4-29-12-6, 104-105 cm, depth $171 \mathrm{~m}$ :

Chiasmolithus grandis, Chiasmolithus staurion, Coccolithus eopelagicus, Discoaster barbadiensis, Sphenolithus furcatolithoides.

4-29-15-1, 68-69 cm, depth $194 \mathrm{~m}$ :

Chiphragmalithus austriacus, Chiphragmalithus mexicanus, Discoaster barbadiensis, Discoaster sp. aff. $D$. colleti.

Lower Middle Eocene

(Discoaster sublodoensis Zone)

4-29-16-1, 140-141 cm, depth $203 \mathrm{~m}$ :

Chiasmolithus sp. [rims], Chiphragmalithus mexicanus, Coccolithus eopelagicus, Discoaster barbadiensis, Discoaster sublodoensis [few].

Age-diagnostic Coccoliths in Selected Samples, Hole 29B

Upper Middle Miocene

(Discoaster hamatus Zone)

4-29B-2B-4, 74-75 cm, depth $73 \mathrm{~m}$ :

Discoaster brouweri, Discoaster calcaris [common] Discoaster challengeri, Discoaster sp. aff. D. hamatus,
Discoaster perclarus, Reticulofenestra pseudoumbilica, Triquetrorhabdulus rugosus.

4-29B-3B-1, 74-75 cm, depth $79 \mathrm{~m}$ :

Discoaster bollii, Discoaster brouweri, Discoaster calcaris, Reticulofenestra pseudoumbilica.

\section{Upper Lower Miocene \\ (Helicopontosphaera ampliaperta Zone)}

4-29B-4B-4, 138-139 cm, depth $93 \mathrm{~m}$ :

Coccolithus eopelagicus, Cyclococcolithus neogammation, Discoaster deflandrei, Discoaster exilis, Helicopontosphaera ampliaperta, Sphenolithus heteromorphus.

\section{Lower Middle (?) Eocene (?Discoaster sublodoenis Zone)}

4-29B-9B-1, 110-111 cm, depth $219 \mathrm{~m}$ :

Discoaster barbadiensis, ?Discoaster sublodoensis, Discasteroides kuepperi.

HOLE 30

(lat $12^{\circ} 52.92^{\prime} \mathrm{N}$., long $63^{\circ} 23.00^{\prime} \mathrm{W}$., depth 1218 meters)

Summary of Coccolith Age Determinations

Coccolith assemblages in sixteen cores from this hole range from upper Pleistocene (Gephyrocapsa oceanica Zone) to lower Miocene (Helicopontosphaera ampliaperta Zone). Pliocene sediment may be present in Cores 7 and 8, but Cores 1 through 6 are all Pleistocene and Cores 9 through 16 are all Miocene. The sediment in Cores 10 through 16 is poorly sorted as to size and may represent slump deposits, although the general sequence of lower to middle Miocene coccolith assemblages is proper order.

Age-diagnostic Coccoliths in Selected Samples, Hole 30

Upper Pleistocene

(Gephyrocapsa oceanica Zone)

4-30-2-4, 75-76 cm, depth $64 \mathrm{~m}$ :

Ceratolithus cristatus, Gephyrocapsa oceanica, Helicopontosphaera kamptneri, Rhabdosphaera clavigera.

Lower Pleistocene

(Coccolithus doronicoides Zone)

4-30-5-1, 74-75 cm, depth $165 \mathrm{~m}$ :

Coccolithus doronicoides, Helicopontosphaera kamptneri, Rhabdosphaera clavigera.

Pliocene to Miocene (Ceratolithus rugosus Zone)

4-30-8-1, 23-24 cm, depth $318 \mathrm{~m}$ :

Ceratolithus rugosus, Ceratolithus tricorniculatus, Discoaster brouweri, Discoaster sp. aff. D. brouweri, Discoaster challengeri, Discoaster pentaradiatus, Discoaster 
surculus, Reticulofenestra pseudoumbilica, Sphenolithus abies.

Lower Upper Miocene

(Triquetrorhabdulus rugosus Zone)

4-30-10-2, 75-76 cm, depth $377 \mathrm{~m}$ :

Discoaster brouweri, Discoaster challengeri, Discoaster sp. aff.D. hamatus, Discoaster pentaradiatus, Discoaster variabilis, Reticulofenestra pseudoumbilica, Sphenolithus abies, Triquetrorhabdulus rugosus.

\section{Middle Miocene \\ (?Discoaster kugleri Zone)}

4-30-12-1, 27-30 cm, depth $393 \mathrm{~m}$ :

Cyclococcolithus leptoporus, Discoaster sp. aff. D. bollii, Discoaster brouweri, Discoaster challengeri, Discoaster subsurculus, Reticulofenestra pseudoumbilica, Triquetrorhabdulus sp. aff. T. rugosus.

\section{Middle Miocene (?Sphenolithus heteromorphus Zone)}

4-30-14-1, 83-85 cm, depth $408 \mathrm{~m}$ :

Coccolithus eopelagicus, Cyclococcolithus leptoporus [rare], Cyclococcolithus neogammation, Discoaster sp. aff. D. bollii, Discoaster variabilis.

Lower Miocene

(Helicopontosphaera ampliaperta Zone)

4-30-15-1, 80-83 cm, depth $413 \mathrm{~m}$ :

Cyclococcolithus neogammation, Discoaster exilis, Sphenolithus heteromorphus.

\section{HOLE 31}

(lat $14^{\circ} 56.60^{\prime} \mathrm{N}$., long $72^{\circ} 01.63^{\prime} \mathrm{W}$., depth 3369 meters)

\section{Summary of Coccolith Age Determinations}

Sediment recovered from Hole 31 (10 cores) ranges from upper Pleistocene (Gephyrocapsa oceanica Zone) to the Oligocene (basal Triquetrorhabdulus carinatus Zone). Lower Pleistocene, lower Pliocene, upper Miocene, and most of the lower Miocene are missing.
Age-diagnostic Coccoliths in Selected Samples, Hole 31

Upper Pleistocene

(Gephyrocapsa oceanica Zone)

4-31-1-5, 74-75 cm, depth $7 \mathrm{~m}$ :

Ceratolithus cristatus, Gephyrocapsa oceanica, Rhabdosphaera clavigera, Scapholithus fossilis.

\section{Upper Pliocene \\ (Discoaster brouweri Zone)}

4-31-1, 75-76 cm, depth $61 \mathrm{~m}$ :

Cyclococcolithus leptoporus var. A, Discoaster brouweri, Discoaster pentaradiatus [rare], Rhabdosphaera clavigera.

4-31-7-1, 66-67 cm, depth $98 \mathrm{~m}$ :

Ceratolithus rugosus, Cyclococcolithus leptoporus var. A, Discoaster brouweri, Discoaster pentaradiatus.

\section{Middle Miocene \\ (?Catinaster coalitus Zone)}

4-31-8-1, 74-75 cm, depth $156 \mathrm{~m}$ :

Cyclococcolithus leptoporus, Discoaster bollii, Discoaster brouweri, Reticulofenestra pseudoumbilica, Triquetrorhabdulus sp. aff. T. rugosus.

\section{Miocene to Oligocene \\ (Triquetrorhabdulus carinatus Zone)}

4-31-9-2, 1-4 cm, depth $214 \mathrm{~m}$ :

Cyclococcolithus neogammation, Discoaster deflandrei, Triquetrorhabdulus carinatus.

\section{Oligocene \\ (basal Triquetrorhabdulus carinatus Zone)}

4-31-10-5, 0-3 cm, depth $276 \mathrm{~m}$ :

Coccolithus sp. aff. C. bisectus, Cyclococcolithus neogammation, Discoaster deflandrei, Sphenolithus ciperoensis [rare], Triquetrorhabdulus carinatus.

\section{REFERENCE}

Bukry, D. and Bramlette, M. N., 1970. Coccolith age determinations Leg 3, Deep Sea Drilling Project. In Maxwell, A. E. et al., 1970, Initial Reports of the Deep Sea Drilling Project. Volume III, Washington (U. S. Government Printing Office). p. 5890. 